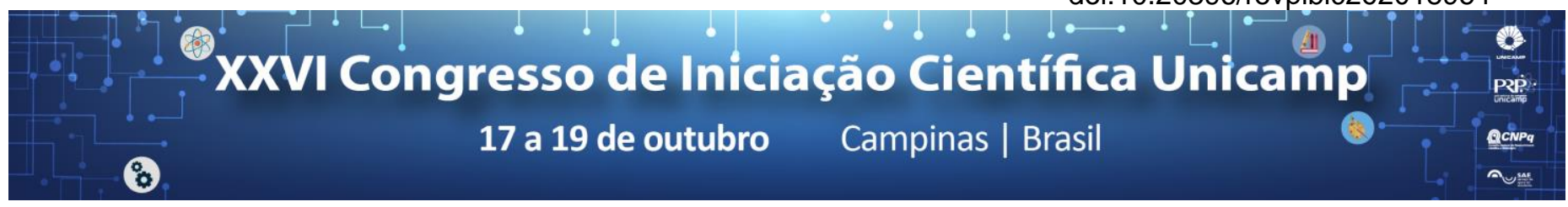

\title{
Polymer Flooding for Enhanced Oil Recovery
}

\author{
Marcelo E. N. Freire Filho*, Rosângela B. Z. L. Moreno
}

\begin{abstract}
In this work, Enhanced Oil Recovery methods were studied, with a focus on polymer flooding. Several works were surveyed to verify which parameters most affect the rheological behaviour of polymer solutions. Characteristics of reservoir rocks, important definitions of the oil industry and mathematical models of the rheology of viscous fluids were investigated as well.
\end{abstract}

\author{
Key words: \\ Oil Recovery, Polymer Flooding, Rheology
}

\section{Introduction}

Oil recovery is divided into three stages: primary, secondary and tertiary. In the first process, the pressure within the reservoir provides the energy necessary to remove the oil. The second stage involves the injection of water or gas into the reservoir to sweep the oil. Subsequently, a third procedure may be applied and its known as tertiary recovery or Enhanced Oil Recovery (EOR). At this stage, special fluids, such as polymer or surfactant solutions, are flooded into the rock. They are designed to increase the viscosity of the injected fluid or to reduce the interfacial tension between them and the oil.

Among the EOR methods, polymer flooding is one of the most used, because it provides good recovery at relatively low cost. Its purpose is to improve the mobility ratio between the displacing fluid and the oil, increasing the sweep efficiency and mitigating adverse effects, such as viscous fingering. Polymer screening criteria include the lithology, temperature, salinity, and permeability of the reservoir rock and the designing-process focused on the ability of the polymer to increase viscosity at a minimum concentration.

Therefore, studying the rheology of the polymer solutions is crucial to make sure that the desired viscosity will be achieved by taking into consideration the characteristics of the target reservoir where the solution is going to be applied. Rock properties were reviewed to assess their impact on the performance of oil recovery using polymers. The rheology of viscous fluids was also surveyed, as well as the most relevant experimental procedures regarding polymer flooding.

\section{Results and Discussion}

Porosity is a measure of the storage capacity of a rock and it is defined as the ratio between the pore and bulk volumes. The interconnected volume (effective porosity) can be determined by gas expansion methods, and its value is closely related to permeability. Permeability is the ability of porous media to let fluids flow through their pores and it can be obtained in core flooding experiments through the Darcy's Law.

Reservoirs are classified in EOR as Sandstones and Carbonates. These categories differ mainly in composition and homogeneity. Carbonates are more heterogeneous and present high permeability zones (due to natural fractures), which makes its field exploration more complicated. They hold about half of the oil and gas of the world, but most of the chemical EOR field projects are carried out in Sandstones.

Viscous fluids are classified as Newtonian and non-Newtonian. For the first one, the relation between shear stress and shear rate is linear. Non-Newtonian fluids present a non-linear relation between those parameters because of the viscosity changes with the shear rate. The simplest model used to describe non-Newtonian fluids is the Power Law.

Viscosity is measured in rheometers or viscometers using different sets of geometries. The most common are the capillary, plate and cone, parallel plates and concentric cylinders or Couette.

Polymer flooding consists of adding polymer, usually in powder, to water or brine, obtaining a solution with higher viscosity. The expectation is to obtain 15 to 20 $\%$ oil incremental recovery over water flooding. This EOR method enhances oil recovery through mechanisms such as the improvement of the mobility ratio between water and oil, the mitigation of viscous fingering, and the reduction of the inject and the produced water.

The most used polymers in EOR are partially hydrolyzed polyacrylamide (HPAM) and Xanthan. HPAM is a synthetic polymer that shows a flexible coil structure, what leads to a higher sensibility to salinity, temperature and mechanical degradation. The degree of hydrolysis has a significant impact on the properties of polyacrylamides. Xanthan, on the other hand, is a biopolymer that presents a rod-like structure and, therefore, is less sensitive to the same parameters. Polymers interact with the porous media and may be retained by adsorption, mechanical entrapment, and hydrodynamic retention.

\section{Conclusions}

The properties of sedimentary rocks were reviewed, which includes porosity and permeability as most relevant for EOR. The rheology of viscous fluids, such as polymer solutions, was assessed concerning the effects of temperature, salinity, and shear rate. Moreover, the experimental procedures used to evaluate rheological parameters were also surveyed.

${ }^{1}$ SORBIE, K. S. Polymer-Improved Oil Recovered. Springer Science \& Business Media, 1991

${ }^{2}$ LUCIA, F. J. Carbonate Reservoir Characterization: An Integrated Approach. Springer, 2007 